\title{
Session V
}

\section{Evolution of light elements in the Universe}




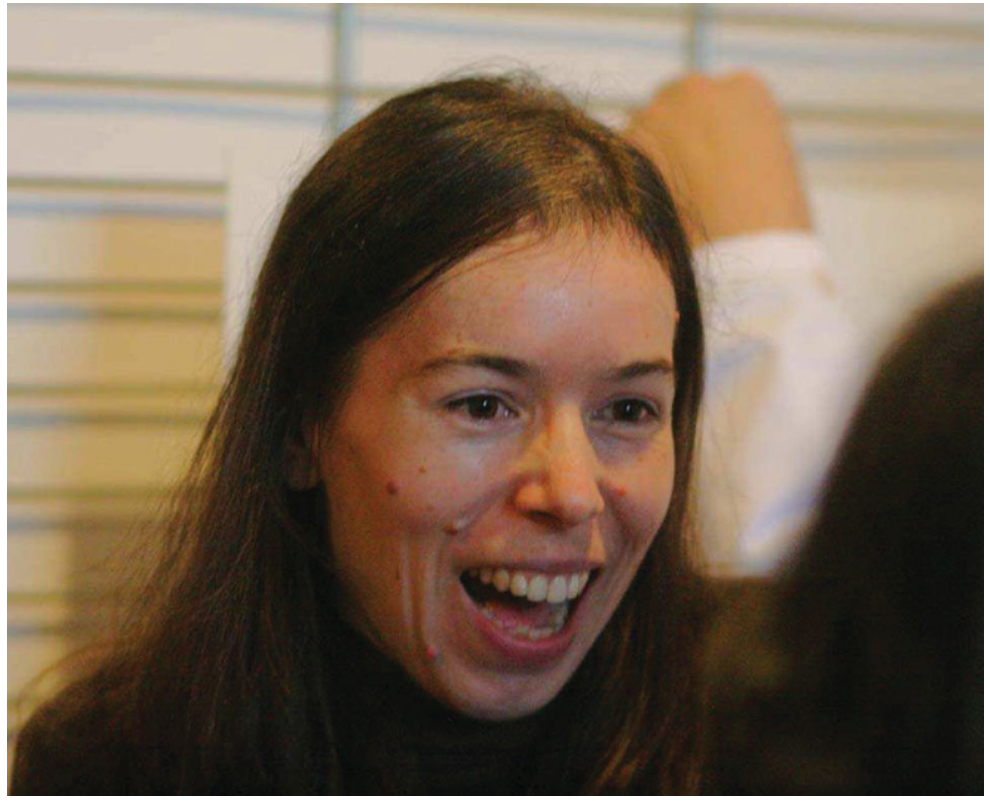

Donatella Romano

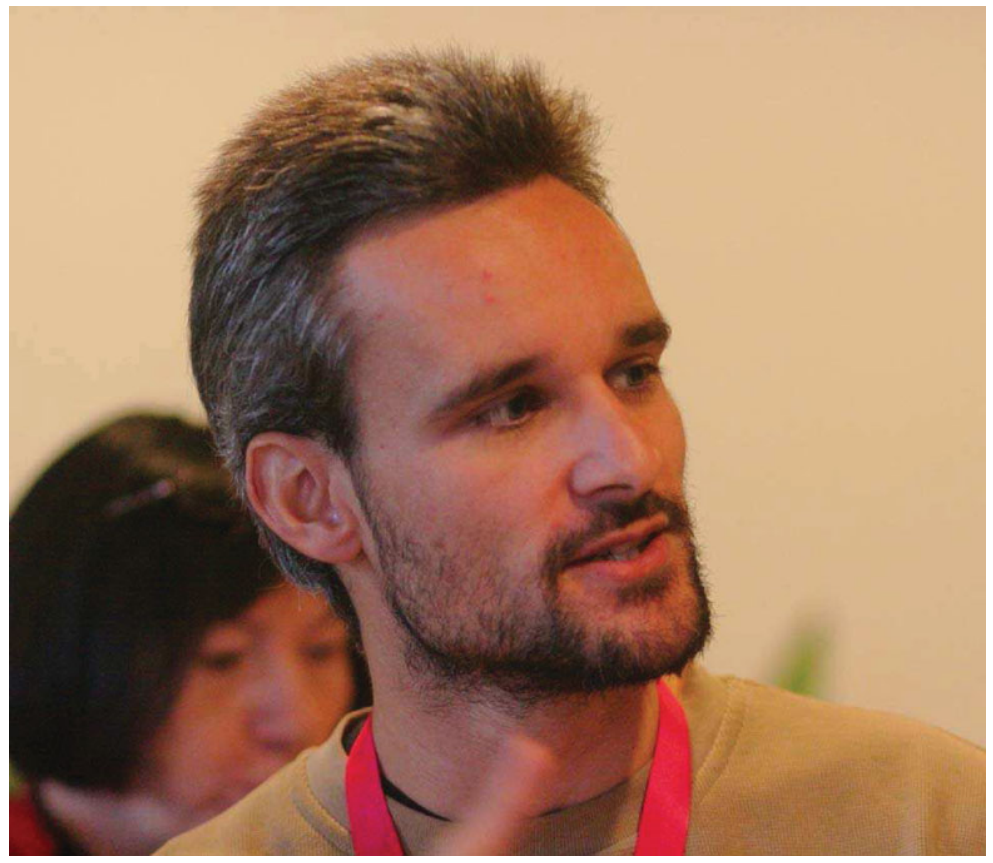

Patrick Eggenberger 


\title{
Galactic evolution of $\mathrm{D},{ }^{3} \mathrm{He}$ and ${ }^{4} \mathrm{He}$
}

\author{
Donatella Romano \\ Dept. of Astronomy, Bologna University, \\ Via Ranzani 1, I-40127, Bologna, Italy \\ and \\ INAF-Bologna Observatory, \\ Via Ranzani 1, I-40127, Bologna, Italy \\ email: donatella.romano@oabo.inaf.it
}

\begin{abstract}
The uncertainties which still plague our understanding of the evolution of the light nuclides D, ${ }^{3} \mathrm{He}$ and ${ }^{4} \mathrm{He}$ in the Galaxy are described. Measurements of the local abundance of deuterium range over a factor of 3 . The observed dispersion can be reconciled with the predictions on deuterium evolution from standard Galactic chemical evolution models, if the true local abundance of deuterium proves to be high, but not too high, and lower observed values are due to depletion onto dust grains. The nearly constancy of the ${ }^{3} \mathrm{He}$ abundance with both time and position within the Galaxy implies a negligible production of this element in stars, at variance with predictions from standard stellar models which, however, do agree with the (few) measurements of ${ }^{3} \mathrm{He}$ in planetary nebulae. Thermohaline mixing, inhibited by magnetic fields in a small fraction of low-mass stars, could in principle explain the complexity of the overall scenario. However, complete grids of stellar yields taking this mechanism into account are not available for use in chemical evolution models yet. Much effort has been devoted to unravel the origin of the extreme helium-rich stars which seem to inhabit the most massive Galactic globular clusters. Yet, the issue of ${ }^{4} \mathrm{He}$ evolution is far from being fully settled even in the disc of the Milky Way.
\end{abstract}

Keywords. Galaxy: abundances, Galaxy: evolution, nuclear reactions, nucleosynthesis, abundances

\section{Introduction}

The discovery of the cosmic microwave background (CMB) by Penzias \& Wilson (1965) in the mid sixties set the stage for a quantitative exploitation of the Big Bang nucleosynthesis theory. In their pioneer studies, Peebles (1966) and Wagoner et al. (1967) demonstrated that D, ${ }^{3} \mathrm{He}$ and ${ }^{4} \mathrm{He}$ could well have been produced in solar-system abundances in the 'primordial fireball'.

In the framework of the standard Big Bang nucleosynthesis (SBBN) theory, the baryonto-photon ratio, $\eta$, is the only parameter regulating the amounts of $\mathrm{D},{ }^{3} \mathrm{He}$ and ${ }^{4} \mathrm{He}$ which emerge from the hot, early Universe (see Fig. 1 and contribution by G. Steigman, this volume). In the nineties, observations, seeking to constrain the primordial abundances of $\mathrm{D},{ }^{3} \mathrm{He}$ and ${ }^{4} \mathrm{He}$ - and, hence, the value of $\eta$ - by probing the most metal-poor environments in the Universe, did not come up with consistent results. Both high and low values were suggested for the primordial deuterium abundance as measured in highredshift, low-metallicity quasar absorption-line systems (QSOALS), $(\mathrm{D} / \mathrm{H})_{\mathrm{P}}=2.5 \times 10^{-4}$ (Carswell et al. 1994, Songaila et al. 1994) or a few times $10^{-5}$ (Burles \& Tytler 1998a,b). Similarly, both low and high values were suggested for the primordial ${ }^{4} \mathrm{He}$ abundance, e.g., $Y_{\mathrm{P}}=0.234 \pm 0.002$ (Olive et al. 1997) or $0.244 \pm 0.002$ (Izotov \& Thuan 1998).

The difficulty to determine $(\mathrm{D} / \mathrm{H})_{\mathrm{P}}$ from observations led to turn the problem upside down and try to infer that quantity by using Galactic chemical evolution (GCE) models. 


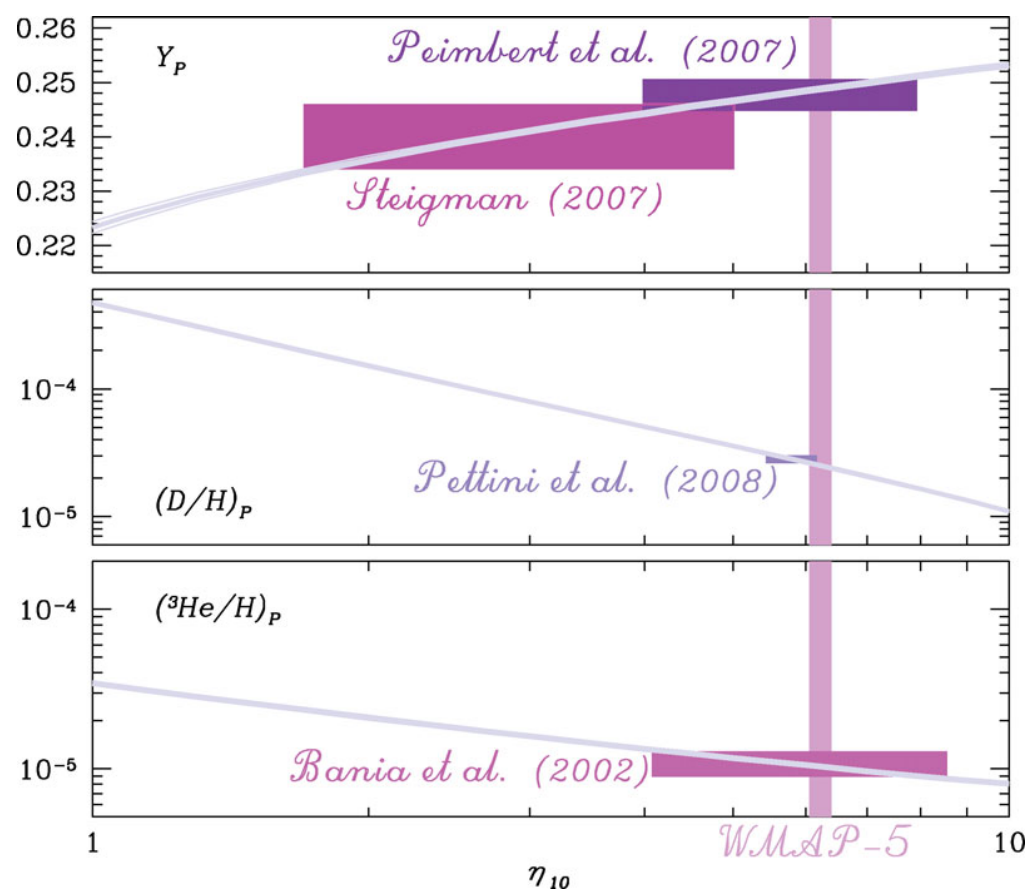

Figure 1. SBBN-predicted primordial mass fraction of ${ }^{4} \mathrm{He}\left(Y_{\mathrm{P}}\right)$ and abundances of $\mathrm{D}$ and ${ }^{3} \mathrm{He}$ (relative to hydrogen by number), as functions of the $\eta_{10}$ parameter, $\eta_{10} \equiv 10^{10}\left(n_{\mathrm{B}} / n_{\gamma}\right)$. Theoretical predictions are from Hata et al. (1995), as updated by G. Steigman (courtesy of G. Steigman). The widths of the curves reflect the uncertainties in the nuclear and weak-interaction rates. The vertical band crossing all panels corresponds to the $\eta_{10}$ value derived from analysis of five-years WMAP data on the CMB anisotropy (Dunkley et al. 2009). Also shown are the most recent estimates of the primordial abundances of $\mathrm{D},{ }^{3} \mathrm{He}$ and ${ }^{4} \mathrm{He}$ from observations, along with the allowed ranges of values for $\eta_{10}$ (boxes; Bania et al. 2002, Peimbert et al. 2007, Steigman 2007, Pettini et al. 2008).

GCE models put stringent limits on the degree of astration suffered by deuterium in the solar vicinity over a Hubble time. Assuming that the local pre-solar (Geiss \& Reeves 1972, Geiss \& Gloeckler 1998) and current (Linsky 1998) D abundances are reasonably well known, they could settle tight limits to the primordial deuterium abundance, and definitively ruled out a high primordial deuterium.

Modelling the Galactic evolution of deuterium is a straightforward task. Since D is completely destroyed as gas cycles through stars and there are no known sources of substantial production other than BBN (Epstein et al. 1976, Prodanović \& Fields 2003), its evolution is obtained for free from GCE models. Good models for the solar neighbourhood - i.e., models which satisfy the majority of the observational constraints available for the solar neighbourhood - have always predicted astration factors $f_{\mathrm{D}} \equiv(\mathrm{D} / \mathrm{H})_{\mathrm{P}} /(\mathrm{D} / \mathrm{H})_{\mathrm{LISM}}$ not in excess of 2-3 for deuterium (Audouze \& Tinsley 1974, Steigman \& Tosi 1992, Edmunds 1994, Galli et al. 1995, Prantzos 1996, Tosi et al. 1998, Chiappini et al. 2002, Romano et al. 2003, 2006). Attempts to accommodate larger astration factors (Vangioni-Flam et al. 1994, Scully et al. 1997) have resulted in models which failed to reproduce important observational constraints. Moreover, the more D is burnt in the Galaxy, the more ${ }^{3} \mathrm{He}$ is produced. Since the abundance of ${ }^{3} \mathrm{He}$ is observed to stay rather constant with both time and position in the Milky Way (Bania et al. 2002), GCE models which overproduce this isotope must be discarded. 

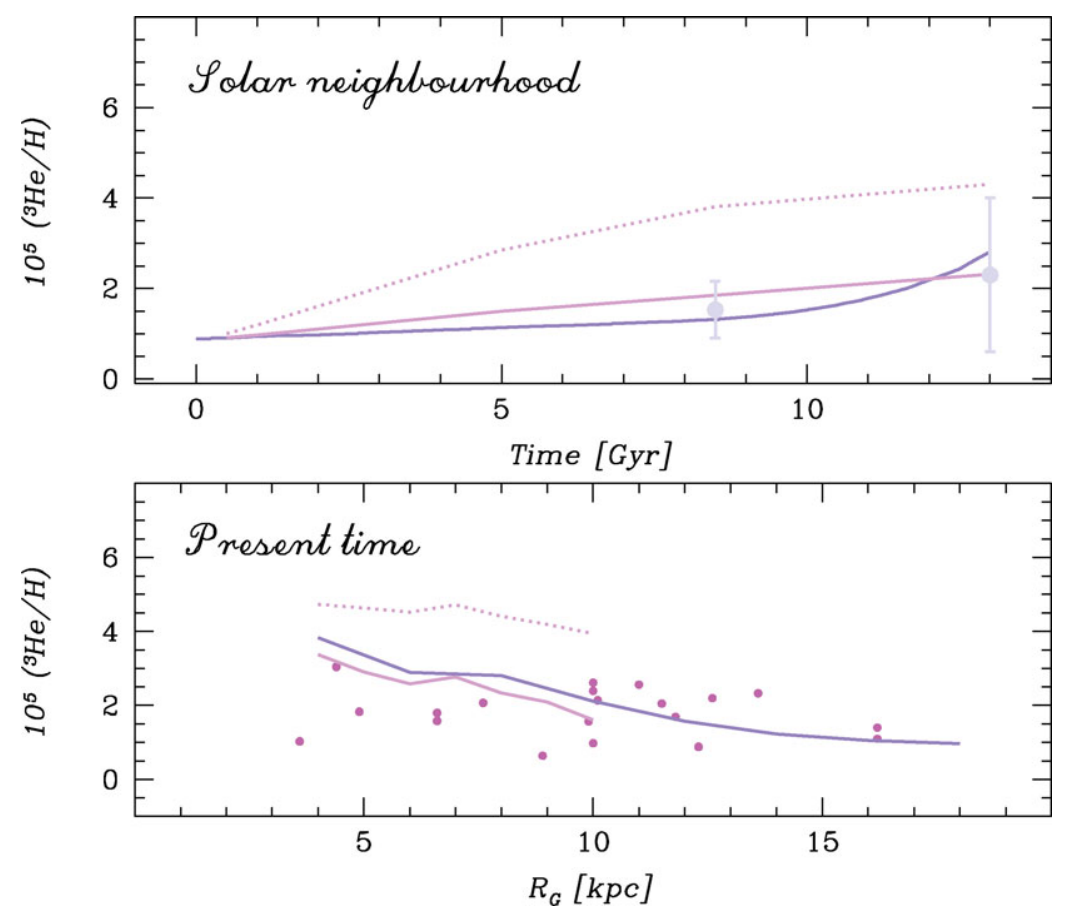

Figure 2. Evolution of ${ }^{3} \mathrm{He} / \mathrm{H}$ in the solar neighbourhood (upper panel) and distribution of ${ }^{3} \mathrm{He} / \mathrm{H}$ across the Galactic disc at the present time (lower panel) for different GCE models, assuming either $(\mathrm{D} / \mathrm{H})_{\mathrm{P}}=2.5 \times 10^{-5}$ (solid lines) or $(\mathrm{D} / \mathrm{H})_{\mathrm{P}}=20 \times 10^{-5}$ (dotted lines). All models assume zero net production of ${ }^{3} \mathrm{He}$ from $93 \%$ of low-mass stars $\left(1-2 \mathrm{M}_{\odot}\right)$ in order to fit the observations (filled circles; upper panel: local pre-solar and current values from Geiss \& Gloeckler 1998; lower panel: H II region abundances from Bania et al. 2002). Figure adapted from Romano et al. (2003).

As first recognized by Truran \& Cameron (1971), GCE models adopting standard prescriptions for the synthesis of ${ }^{3} \mathrm{He}$ in stars dramatically overestimate its abundance in the Milky Way (see also Rood et al. 1976). According to standard stellar models, ${ }^{3} \mathrm{He}$ is most efficiently produced on the main sequence (MS) of 1-2 $\mathrm{M}_{\odot}$ stars through the action of the p-p chains. In order not to overproduce ${ }^{3} \mathrm{He}$ in the course of Galactic evolution, it has become customary to assume that some unknown ${ }^{3} \mathrm{He}$-destruction mechanism is at work in more than 90\% of low-mass stars (Dearborn et al. 1996, Galli et al. 1997, Chiappini et al. 2002, Romano et al. 2003). Hogan (1995) and Charbonnel (1995) have suggested 'extra mixing' during the red giant branch (RGB) phase of low-mass stars as a possible solution (see also Charbonnel \& Do Nascimento 1998, Sackmann \& Boothroyd 1999). In Fig. 2, we compare the predictions of two successful models for the chemical evolution of the Milky Way (the one by Chiappini et al. 2002 and Model 1 of Tosi 1988) to ${ }^{3} \mathrm{He}$ data for the solar neighbourhood (upper panel) and the Galactic disc (lower panel). Despite different assumptions about the infall law, star formation rate and stellar initial mass function (IMF), both models need to assume that at least $93 \%$ of low-mass stars burn the ${ }^{3}$ He they have produced on the MS in later evolutionary phases in order to fit the observations. It is worth noticing that the good agreement between model predictions and observations depends also on the adopted value of the primordial deuterium abundance: if $(\mathrm{D} / \mathrm{H})_{\mathrm{P}}=20 \times 10^{-5}$, rather than $2.5 \times 10^{-5}$ (dotted versus solid lines in Fig. 2), both the local behaviour of ${ }^{3} \mathrm{He}$ with time and its present distribution across the Galactic disc 
Table 1. Abundances of D, ${ }^{3} \mathrm{He}$ and ${ }^{4} \mathrm{He}$ at different epochs

\begin{tabular}{cccccc}
\hline Nuclide & Units & $\begin{array}{c}\text { SBBN+WMAP } \\
(\mathbf{1 3 . 7} \text { Gyr ago })\end{array}$ & $\begin{array}{c}\text { Low- } \boldsymbol{Z} \text { systems } \\
(\mathbf{1 0}-\mathbf{1 3} \text { Gyr ago })\end{array}$ & $\begin{array}{c}\text { Pre-solar matter } \\
(\mathbf{4 . 5} \text { Gyr ago })\end{array}$ & $\begin{array}{c}\text { LISM } \\
\text { (Today) }\end{array}$ \\
\hline $\mathrm{D}$ & $10^{5}(\mathrm{D} / \mathrm{H})$ & $2.49 \pm 0.17^{(1)}$ & $2.8 \pm 0.2^{(2)}$ & $2.1 \pm 0.5^{(3)}$ & $2.31 \pm 0.24^{(4)}$ \\
& & & & $0.98 \pm 0.19^{(5)}$ \\
& & & & $2.0 \pm 0.1^{(6)}$ \\
${ }^{3} \mathrm{He}$ & $10^{5}\left(^{3} \mathrm{He} / \mathrm{H}\right)$ & $1.00 \pm 0.07^{(1)}$ & $1.1 \pm 0.2^{(7)}$ & $1.5 \pm 0.2^{(3)}$ & $2.4 \pm 0.7^{(8)}$ \\
${ }^{4} \mathrm{He}$ & $Y$ & $0.2486 \pm 0.0002^{(1)}$ & $0.2477 \pm 0.0029^{(9)}$ & $0.2703^{(11)}$ & \\
& & & $0.240 \pm 0.006^{(10)}$ & & \\
\hline
\end{tabular}

Notes:

${ }^{a}$ Using $\eta_{10}=6.23 \pm 0.17$ from analysis of 5 -years WMAP data (Dunkley et al. 2009).

References:

(1) Cyburt et al. (2008); (2) Pettini et al. (2008); (3) Geiss \& Gloeckler (1998); (4) Linsky et al. (2006); (5)

Hébrard et al. (2005); (6) Prodanović et al. (2009); (7) Bania et al. (2002); (8) Gloeckler \& Geiss (1996); (9)

Peimbert et al. (2007); (10) Steigman (2007); (11) Asplund et al. (2009).

can not be reproduced by the models, independently of how many low-mass stars burn their ${ }^{3} \mathrm{He}$ on the RGB.

As far as ${ }^{4} \mathrm{He}$ is concerned, there has been a general consensus that the relative heliumto-metal enrichment ratio in the solar neighbourhood is $\Delta Y / \Delta Z \sim 2$, both from a theoretical (Chiosi \& Matteucci 1982, Maeder 1992, Chiappini et al. 2003) and an observational point of view (e.g., Casagrande et al. 2007). Yet, hints for very different values of this ratio were reported early on in the literature (Danziger 1970, and references therein).

The determination of the parameter $\eta$ from WMAP data (see text by J. Dunkley, this volume) has allowed to fix, with unprecedented precision, the primordial abundances of the light elements in the framework of the SBBN model (see Cyburt et al. 2008 for recent work). The primordial abundances of $\mathrm{D},{ }^{3} \mathrm{He}$ and ${ }^{4} \mathrm{He}$ determined indirectly from the CMB anisotropies agree very well with those inferred from recent, direct observations (see Fig. 1 and Table 1), although one must be aware that the latter actually provide only lower/upper limits to the true primordial abundances. Above all, it is clear that the determination of the primordial abundance of deuterium is converging towards a low value, beautifully confirming earlier findings from GCE models.

In the following sections, the remaining (major) causes of uncertainty, which hamper our current understanding of the Galactic chemical evolution of the light elements D, ${ }^{3} \mathrm{He}$ and ${ }^{4} \mathrm{He}$, are discussed, element by element.

\section{Deuterium}

The joint determinations of the primordial and pre-solar deuterium abundances (Table 1) point to a small depletion of deuterium from the Big Bang up to the solar system formation 4.5 Gyr ago. However, the present-day abundance of deuterium in the solar vicinity is currently under debate. The FUSE satellite has measured the deteurium abundance along the lines of sight to several stars in the Local Bubble as well as beyond it, up to 1-2 kpc away. The dispersion (by a factor of 3) which has been found in the measurements (Linsky et al. 2006) makes it hard to interpret the data in the context of standard GCE models. It has been suggested (Hébrard et al. 2005, Linsky et al. 2006) that either the lowest (see also text by G. Hébrard, this volume) or the highest (see also text by J. Linsky, this volume) observed abundances are indicative of the actual value of the deuterium abundance in the local ISM (LISM). However, neither of these values can be reproduced by GCE models in agreement with all the major observational constraints for the solar neighbourhood (Romano et al. 2006). Very recently, using a Bayesian analysis approach, Prodanović et al. (2009) have provided another estimate of the true LISM 
deuterium abundance, which places it very close to the $\mathrm{D}$ abundance at the time of the formation of the Sun (see Table 1).

In Fig. 3 we show the evolution of deuterium in the solar vicinity and the deuterium abundance profile across the Milky Way disc. The adopted GCE model, from Romano et al. (2006), is the one which allows for the lowest D astration factor in the solar vicinity $\left(f_{\mathrm{D}}=1.39\right)$. Here it has been recomputed assuming $(\mathrm{D} / \mathrm{H})_{\mathrm{P}}=2.8 \times 10^{-5}$ rather than $2.6 \times 10^{-5}$ as in Romano et al. (2006). Extrapolation of the theoretical results towards the Galaxy center has been performed by taking into account the detailed results of a model for the Galactic bulge by Matteucci et al. (1999). Data are from Table 1 for local values (at a radius $R_{\mathrm{G}}=8 \mathrm{kpc}$ ), from Rogers et al. (2005) for the outer disc and from Lubowich et al. (2000) for a region at $10 \mathrm{pc}$ distance from the Galactic center.

The agreement of the model predictions with the data is striking, especially if the true value for the local abundance of deuterium is the one suggested by Prodanovic et al. (2009; see also contribution by T. Prodanović, this volume). In this context, lower observed local abundances of deuterium would be due to $\mathrm{D}$ depletion onto dust grains (Linsky et al. 2006, Steigman et al. 2007, and references therein). The highest observed local $\mathrm{D}$ values are marginally consistent with the estimate of the true $\mathrm{D}$ value by Prodanović et al. (2009).
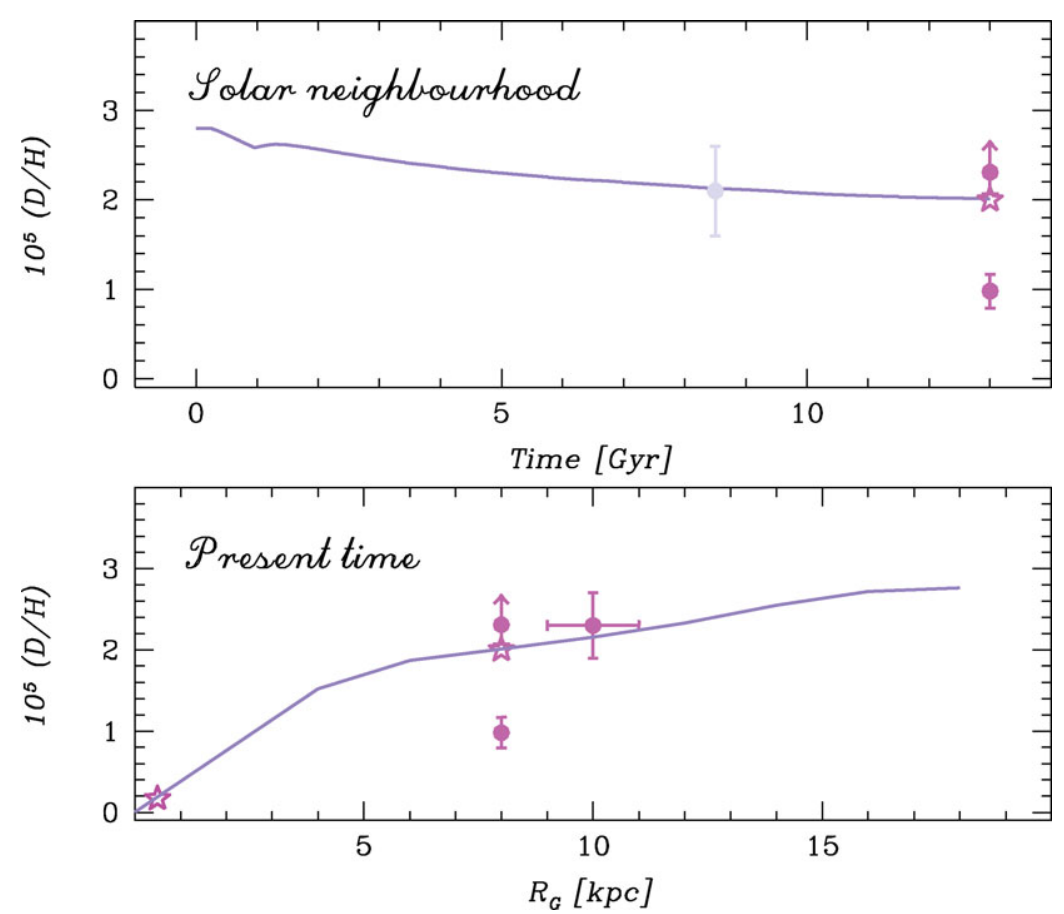

Figure 3. Evolution of $\mathrm{D} / \mathrm{H}$ in the solar neighbourhood (upper panel) and distribution of $\mathrm{D} / \mathrm{H}$ across the Galactic disc at the present time (lower panel) for the GCE model (solid lines in both panels) of Romano et al. (2006) with the lowest $\mathrm{D}$ astration factor, assuming $(\mathrm{D} / \mathrm{H})_{\mathrm{P}}=2.8 \times$ $10^{-5}$. Data are from Table 1 for local values, from Rogers et al. (2005) for the outer disc (filled circle at $R_{\mathrm{G}}=10 \pm 1 \mathrm{kpc}$, bottom panel) and from Lubowich et al. (2000) for the inner Galaxy (star at $R_{\mathrm{G}}=10 \mathrm{pc}$, bottom panel). 


\section{Helium-3}

As far as ${ }^{3} \mathrm{He}$ is concerned, we must recognize that the problems raised in pioneering works by people such as Truran \& Cameron (1971), Reeves et al. (1973) and Tinsley (1974) (to name a few) are still unsolved: we must postulate that some unknown ${ }^{3} \mathrm{He}-$ destruction mechanism is at work in not less than $90 \%$ of low-mass stars in order not to overproduce ${ }^{3} \mathrm{He}$ in the course of Galactic evolution (see discussion in Sect. 1). But which is the physical mechanism responsible for that? In a coherent picture, one must also be able to explain the existence of a few planetary nebulae $(\mathrm{PNe})$ with high ${ }^{3} \mathrm{He}$ content, consistent with predictions from standard stellar models (e.g., Balser et al. 1999). Recently, the inclusion of thermohaline mixing in detailed stellar evolutionary models (Charbonnel \& Zahn 2007) has shown that this is likely to be the 'extra mixing' mechanism we have been searching for years. Indeed, thermohaline mixing is able to efficiently destroy ${ }^{3} \mathrm{He}$ on the RGB of low-mass $\left(1-2 \mathrm{M}_{\odot}\right)$ stars, when not inhibited by magnetic fields. Details on this interesting process can be found in the contribution by N. Lagarde and C. Charbonnel to these conference proceedings (see also text by R. Stancliffe, this volume). The expected output of stellar models including thermohaline mixing are complete grids of yields. New GCE models computed with such new yields will hopefully lead the so-called ${ }^{3} \mathrm{He}$ problem to an end.

\section{Helium-4}

As far as ${ }^{4} \mathrm{He}$ is concerned, there are many open issues to be discussed.

First of all, it is worth stressing that, when dealing with the evolution of ${ }^{4} \mathrm{He}$ in the Galactic disc, we can rely on only a few data (see also M. Peimbert et al., this volume), namely, the abundance of ${ }^{4} \mathrm{He}$ in the Sun at the time of its birth, which is by now quite well established (Asplund et al. 2009), the abundance of ${ }^{4} \mathrm{He}$ in M 17, an H II region located in the inner Galaxy, and the helium-to-metal enrichment ratio as derived from nearby K-dwarf stars. The latter quantity, however, is affected by a rather large error and can only be trusted around solar metallicity (see Casagrande et al. 2007 and contribution by L. Casagrande, this volume).

Fig. 4 shows the behaviour of $Y$ as a function of metallicity $\left[10^{6}(\mathrm{O} / \mathrm{H})\right]$ in the solar neighbourhood, as predicted by the two-infall model of Chiappini et al. (1997). The model has been computed with different prescriptions on the stellar yields and primordial mass fraction of ${ }^{4} \mathrm{He}$ (see figure caption for details). Although the models adopting the ${ }^{4} \mathrm{He}$ yields from rotating, mass-losing stellar models (Fig. 4, dotted and dashed lines) provide a good fit to the available solar neighbourhood data, performing definitely better than the model using stellar yields without stellar rotation and mass loss (Fig. 4, solid line), it has to be reminded that the data for M 17 cannot be satisfactorily reproduced by any GCE model yet (see M. Peimbert et al., this volume, their figure 1).

Another debated topic is the extreme He enhancement in Galactic globular clusters (GCs). The presence of multiple MSs in the GCs $\omega$ Cen and NGC 2808 (Piotto et al. 2005, 2007), indeed, is most convincingly explained in terms of an extreme He enhancement of the bluest MS population (Norris 2004). Helium abundances as high as $Y \sim 0.4$ are suggested, which for $\omega$ Cen imply a helium-to-metal enrichment ratio $\Delta Y / \Delta Z \geqslant 70$ (Piotto et al. 2005). Such a value is outstandingly larger than that quoted for the solar neighbourhood around and above solar metallicity from a sample of nearby K-dwarf stars, $\Delta Y / \Delta Z=2.1 \pm 0.9$ (Casagrande et al. 2007). Attemps have been made to explain such extreme ${ }^{4} \mathrm{He}$ abundances in the framework of two main competing scenarios, the so-called 'asymptotic giant branch (AGB) self-pollution scenario' (P. Ventura, this volume) and 
the so-called 'fast rotating massive star (FRMS) self-pollution scenario' (T. Decressin, this volume). Both scenarios have to reproduce other chemical peculiarities of GC stars besides the 'anomalous' ${ }^{4} \mathrm{He}$ abundances, and both have advantages and disadvantages. As a common limitation, they are presently able to deal only with two-population clusters. However, in $\omega$ Cen - the object for which the most compelling evidence for the need for a huge helium enrichment is found - there is clear-cut evidence of the presence of complex, multiple populations (e.g., Pancino et al. 2000). We (Romano et al. 2010) have recently proposed that the presence of extreme He-rich stars in $\omega$ Cen can be explained in the context of a model where the cluster is the remnant of a much more massive parent system, that evolved in isolation for a relatively long time $-3 \mathrm{Gyr}$, with the bulk of the stars forming during the first 1 Gyr. The system was then captured and partially disrupted by the Milky Way (see contribution by D. Romano et al., this volume). The key ingredient in our model is the development of a differential galactic wind, which selectively removes from the progenitor galaxy mostly the elements restored to the ISM through fast polar winds from massive stars and supernova (SN) explosions. Elements restored to the ISM through gentle winds from both AGB and FRMSs are, instead, mostly retained in the cluster potential well, where they enter the formation of successive

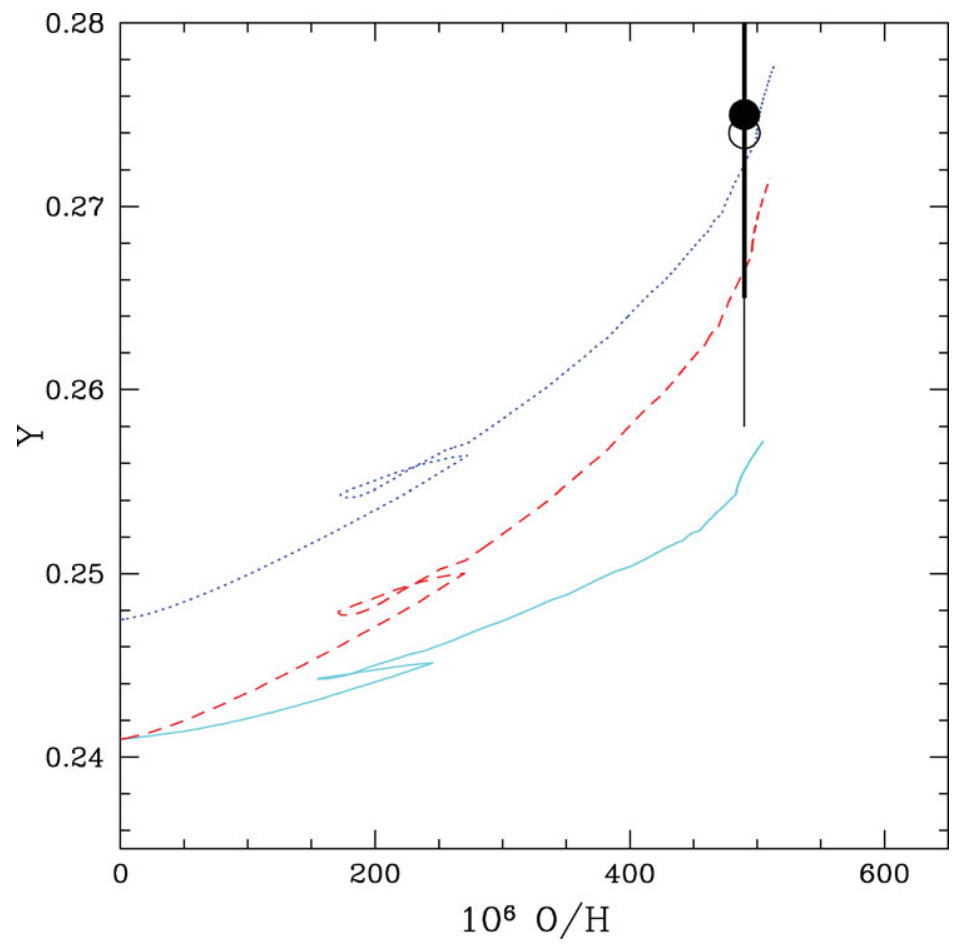

Figure 4. $Y$ versus $10^{6}(\mathrm{O} / \mathrm{H})$ in the solar neighbourhood predicted by the two-infall model of Chiappini et al. (1997) with different prescriptions on the stellar nucleosynthesis. Solid line: the model adopts the van den Hoek \& Groenewegen (1997) yields for low- and intermediate-mass stars and the Woosley \& Weaver (1995) yields for massive stars; dashed line: the model is computed with the yields of Meynet \& Maeder (2002), taking into account the effects of rotation on stellar evolution, for the whole range of stellar masses; dotted line: same model as the previous one, but starting from a higher primordial ${ }^{4} \mathrm{He}$ abundance, $Y_{\mathrm{P}}=0.248$ rather than 0.241 . The model predictions are compared with the solar value (oxygen from Allende-Prieto et al. 2001, $Y$ from Anders \& Grevesse 1989 - open circle with thin errorbar - and Grevesse \& Sauval 1998 - filled circle with thick errorbar). Figure from Chiappini et al. (2003). 
stellar generations. Since, according to the latest stellar evolutionary computations, ${ }^{4} \mathrm{He}$ is dispersed in the ISM by means of low-energy stellar winds by both AGBs and FRMSs, while metals are mostly expelled through SN explosions, a high $\Delta Y / \Delta Z$ is naturally obtained in the framework of our model. Other important observational constraints can also be satisfactorily reproduced.

Since differential galactic winds do, as a matter of fact, modify somewhat arbitrarily the true (effective) yields of the various elements, a better assessment of the stellar yields of ${ }^{4} \mathrm{He}$ is mandatory.

Finally, it is worth reminding that the usually quoted value of $Y \sim 0.4$ for the extreme He-rich GC stars could be revised downwards to as low as $Y \sim 0.3$ (L. Casagrande, this volume).

\section{Conclusions}

We have reviewed the evolution of the light elements D, ${ }^{3} \mathrm{He}$ and ${ }^{4} \mathrm{He}$ in the Milky Way, emphasizing recent developments and open problems. We summarize our conclusions as follows:

(a) GCE models are consistent with the relatively high value of $(\mathrm{D} / \mathrm{H})_{\mathrm{LISM}}=(2.0 \pm$ $0.1) \times 10^{-5}$ suggested by Prodanović et al. (2009) from their Bayesian analysis of FUSE data. Standard GCE models are instead unable to explain values of $(\mathrm{D} / \mathrm{H})_{\text {LISM }}$ significantly higher/lower than this.

(b) The need for some 'extra mixing' to destroy ${ }^{3} \mathrm{He}$ in most $(>90 \%)$ of $1-2 \mathrm{M}_{\odot}$ stars came from GCE arguments more than 30 years ago. The way to the understanding of the physical processes underlying this assumption has been a long one, but now thermohaline mixing seems to be a good candidate to solve the long-standing issue of ${ }^{3} \mathrm{He}$ evolution. Stellar yields taking this mechanism into account are needed for use in GCE models.

(c) It is still debated whether the chemical peculiarities seen in a fraction of Galactic globular cluster stars - first of all an impressive helium enrichment - are due to selfpollution from AGBs or FRMSs. In the very peculiar case of $\omega$ Cen, which likely suffered a complicated star formation history as the nucleus of a larger system, both stellar categories should have polluted the ISM. In such a scenario, the observed chemical 'anomalies' would be driven by the action of differential galactic outflows, venting out preferentially metals. However, it is still unclear if such an extreme scenario could apply to other GCs as well.

\section{Acknowledgements}

I thank the organizers for their kind invitation and for giving me the opportunity to attend such a lively conference. I would like to express my gratitude to Francesca Matteucci and Monica Tosi for advice over the years, and to Johannes Geiss and Gary Steigman for helpful discussions. Generous financial support from IAU is also gratefully acknowledged. The author's research at Bologna University is supported by Italian MIUR under grant PRIN 2007, prot. 2007JJC53X_001.

\section{References}

Allende-Prieto, C., Lambert, D. L., \& Asplund, M. 2001, ApJ, 556, L63

Anders, E. \& Grevesse, N. 1989, Geochim. Cosmochim. Acta, 53, 197

Asplund, M., Grevesse, N., Sauval, A. J., \& Scott, P. 2009, ARA\&A, 47, 481

Audouze, J. \& Tinsley, B. M. 1974, ApJ, 192, 487 
Balser, D., Rood, R. T., \& Bania, T .M. 1999, ApJ, 522, L73

Bania, T. M., Rood, R. T., \& Balser, D. S. 2002, Nature, 415, 54

Burles, S. \& Tytler, D. 1998a, ApJ, 499, 699

Burles, S. \& Tytler, D. 1998b, ApJ, 507, 732

Carswell, R. F., Rauch, M., Weymann, R. J., Cooke, A. J., \& Webb, J. K. 1994, MNRAS, 268, L1

Casagrande, L., Flynn, C., Portinari, L., Girardi, L., \& Jimenez, R. 2007, MNRAS, 382, 1516

Charbonnel, C. 1995, ApJ, 453, L41

Charbonnel, C. \& Do Nascimento, J. D., Jr. 1998, A\&\&A, 336, 915

Charbonnel, C. \& Zahn, J.-P. 2007, A\&A, 467, L15

Chiappini, C., Matteucci, F., \& Gratton, R. 1997, ApJ, 477, 765

Chiappini, C., Matteucci, F., \& Meynet, G. 2003, A\&A, 410, 257

Chiappini, C., Renda, A., \& Matteucci, F. 2002, A\&SA, 395, 789

Chiosi, C. \& Matteucci, F. 1982, A\&GA, 105, 140

Cyburt, R. H., Fields, B. D., \& Olive, K. A. 2008, JCAP, 11, 012

Danziger, I. J. 1970, ARA\&A, 8, 161

Dearborn, D. S. P., Steigman, G., \& Tosi, M. 1996, ApJ, 465, 887

Dunkley, J., et al. 2009, ApJS, 180, 306

Edmunds, M. G. 1994, MNRAS, 270, L37

Epstein, R. I., Lattimer, J. M., \& Schramm, D. N. 1976, Nature, 263, 198

Galli, D., Palla, F., Ferrini, F., \& Penco, U. 1995, ApJ, 443, 536

Galli, D., Stanghellini, L., Tosi, M., \& Palla, F. 1997, ApJ, 477, 218

Geiss, J. \& Gloeckler, G. 1998, Space Sci. Rev., 84, 239

Geiss, J. \& Reeves, H. 1972, A\&SA, 18, 126

Gloeckler, G. \& Geiss, J. 1996, Nature, 381, 210

Grevesse, N. \& Sauval, A. J. 1998, Space Sci. Rev., 85, 161

Hata, N., Scherrer, R. J., Steigman, G., Thomas, D., Walker, T. P., Bludman, S., \& Langacker, P. 1995, Phys. Rev. Lett., 75, 3977

Hébrard, G., Tripp, T. M., Chayer, P., Friedman, S. D., Dupuis, J., Sonnentrucker, P., Williger, G. M., \& Moos, M. W. 2005, ApJ, 635, 1136

Hogan, G. 1995, ApJ, 441, L17

Izotov, Y. I. \& Thuan, T. X. 1998, ApJ, 500, 188

Linsky, J. L. 1998, Space Sci. Rev., 84, 285

Linsky, J. L., et al. 2006, ApJ, 647, 1106

Lubowich, D. A., Pasachoff, J. M., Balonek, T. J., Millar, T. J., Tremonti, C., Roberts, H. \& Galloway, R. P. 2000, Nature, 405, 1025

Maeder, A. 1992, A\& A, 264, 105

Meynet, G. \& Maeder, A. 2002, $A \mathscr{E} A, 390,561$

Matteucci, F., Romano, D., \& Molaro, P. 1999, A\& $A, 341,458$

Norris, J. E. 2004, ApJ, 612, L25

Olive, K. A., Skillman, E., \& Steigman, G. 1997, ApJ, 483, 788

Pancino, E., Ferraro, F. R., Bellazzini, M., Piotto, G., \& Zoccali, M. 2000, ApJ, 534, L83

Peebles, P. J. E. 1966, Phys. Rev. Lett., 16, 410

Peimbert, M., Luridiana, V., \& Peimbert, A. 2007, ApJ, 666, 636

Penzias, A. A. \& Wilson, R. W. 1965, ApJ, 142, 419

Pettini, M., Zych, B. J., Murphy, M. T., Lewis, A., \& Steidel, C. C. 2008, MNRAS, 391, 1499

Piotto, G., et al. 2005, ApJ, 621, 777

Piotto, G., Bedin, L. R., Anderson, J., King, I. R., Cassisi, S., Milone, A. P., Villanova, S., Pietrinferni, A., \& Renzini, A. 2007, ApJ, 661, L53

Prantzos, N. 1996, A\&GA, 310, 106

Prodanović, T. \& Fields, B. D. 2003, ApJ, 597, 48

Prodanović, T., Steigman, G., \& Fields, B. D. 2009, preprint (arXiv:0910.4961)

Reeves, H., Audouze, J., Fowler, W. A., \& Schramm, D. N. 1973, ApJ, 179, 909 
Rogers, A. E. E., Dudevoir, K. A., Carter, J. C., Fanous, B. J., Kratzenberg, E., \& Bania, T. M. 2005, ApJ, 630, L41

Romano, D., Tosi, M., Matteucci, F., \& Chiappini, C. 2003, MNRAS, 346, 295

Romano, D., Tosi, M., Chiappini, C., \& Matteucci, F. 2006, MNRAS, 369, 295

Romano, D., Tosi, M., Cignoni, M., Matteucci, F., Pancino, E., \& Bellazzini, M. 2010, MNRAS, in press (arXiv:0910.1299)

Rood, R. T., Steigman, G., \& Tinsley, B. M. 1976, ApJ, 207, L57

Sackmann, I.-J. \& Boothroyd, A. I. 1999, ApJ, 510, 217

Scully, S., Cassé, M., Olive, K. A., \& Vangioni-Flam, E. 1997, ApJ, 476, 521

Songaila, A., Cowie, L. L., Hogan, C. J., \& Rugers, M. 1994, Nature, 368, 599

Steigman, G. 2007, Annu. Rev. Nucl. Part. Sci., 57, 463

Steigman, G. \& Tosi, M. 1992, ApJ, 401, 150

Steigman, G., Romano, D., \& Tosi, M. 2007, MNRAS, 378, 576

Tinsley, B. M. 1974, ApJ, 192, 629

Tosi, M. 1988, A\& A, 197, 33

Tosi, M., Steigman, G., Matteucci, F., \& Chiappini, C. 1998, ApJ, 498, 226

Truran, J. W. \& Cameron, A. G. W. 1971, Ap\&SS, 14, 179

van den Hoek, L. B. \& Groenewegen, M. A. T. 1997, A\&SAS, 123, 305

Vangioni-Flam, E., Olive, K. A., Prantzos, N. 1994, ApJ, 427, 618

Wagoner, R. V., Fowler, W. A., \& Hoyle, F. 1967, ApJ, 148, 3

Woosley, S. E. \& Weaver, T. A. 1995, ApJS, 101, 181 